\title{
New Working Practices: A Scientometric Review
}

\author{
Olugbenga Timo Oladinrin ${ }^{1 *}$, Wadu Mesthrige Jayantha ${ }^{2}$, Tochukwu Moses ${ }^{1}$ \\ ${ }^{1}$ School of Architecture and Built Environment, University of Wolverhampton, Wolverhampton, United Kingdom \\ ${ }^{2}$ School of Property Construction and Project Management, RMIT University, Melbourne, Australia \\ *Corresponding author's email: o.oladinrin@wlv.ac.uk
}

Article history: Received: 23 January 2021 Received in revised form: 5 March 2021 Accepted: 3 April 2021 Published online: 23 June 2021

\begin{abstract}
Study on New Working Practices (NWPs), which is the subject of this review paper, has created a large body of literature. Studies in this research area are progressing quickly, and it is important to stay abreast of new trends and essential factors in the growth of mutual awareness. This study evaluates the global scientific output of New Working Practices (NWPs) research and explores their hotspots and frontiers from 1980 to 2018 (pre-COVID-19), using bibliometric methods. 850 relevant articles were retrieved from the Web of Science Core Collection (WoSCC) and analysed. Scientometric method and Citespace VI were used to analyse the bibliometric data. Reference citation and cocitation networks were plotted, while keywords were used to analyse the research hotspots and trends. There is a significant increase in the number of annual publications with time. The United Kingdom (UK) ranked highest in the countries with most publications, and the leading author is Friedhelm Nachreiner based on publication counts. The most cited author/organisation is the UK Department of Health. Performance, work, and flexible working are the research hotspots, while flexible working arrangement represents the prominent research domain. The study offers valuable references for researchers, industry practitioners and policymakers.
\end{abstract}

Keywords: CiteSpace, flexible working, new working practices, scientometric, United Kingdom

(C) 2021 Penerbit UTM Press. All rights reserved

\subsection{INTRODUCTION}

Workplace transition is a global phenomenon. The essence of employment is shifting, and the population's makeup is also adapting to change (Waterhouse et al., 1999). Literature has demonstrated that businesses adapt to the changing world of work, while workplaces begin to undergo shifts in the societal, technological, and economic sense (Askenazy et al., 2002; Bell \& Anderson, 1999; McEwan \& Ennals, 2005; Tucker et al., 2014). This change is often engineered by the growth and extensive use of information technology (IT) and the gravitation of a manufacturing-based economy towards a service-based economy (McOrmond, 2004). In addition to the progression of IT, organisational theorists have found out that rising pressures on business institutions to be more innovative, sensitive, and customer-focused are another driver that leads to shifting patterns (Heerwagen et al., 2016). Since the early 1990s, many corporations, particularly in advanced economies, have begun to accept new working practices (NWPs), with nearly two-thirds of large organisations globally adopting NWPs (Dixon \& Ross, 2011). NWPs are innovative or flexible ways of working, typically comprising four key components viz: teambased work; job autonomy; job rotation; and interaction/communication in the workplace (Ollo-Lopez et al., 2010; Osterman, 1994). Consequently, the current pandemic of COVID-19 is having a tremendous influence on organisations around the world, with companies changing the way work is done (Bailey \& Breslin, 2021).To accommodate such changes, two-thirds of large organisations, especially in the West have embraced NWPs (Dixon \& Ross, 2011), such as team-working, teleworking, hot-desking, activity-based-working, and home-working (Delanoeije \& Verbruggen, 2019; Dixon, 2005; Haynes, 2008). These are new practices that promote developing partnerships between company teams, promoting a culture of cooperation, recruiting and maintaining the best talent, improving competitiveness, and stimulating creativity. Jayantha et al. (2007) posited that the most important driver of NWPs is 'space costs'. As digital technologies continue to drive the way people work in workplaces, NWPs, such as task rotation, collaboration, teleworking, are gaining ground. The advent of NWPs has raised concerns about hierarchical structure and power in terms of mobility, transparency, hierarchy, and cooperation. However, the lack of clarification in the area of NWPs is a big concern due to various names and definitions.

A cross-country overview revealed that; NWPs occur in clusters (i.e. some NWPs happen together in the same place), leading to an increase in productivity, and are supported by high skills (Eriksson, 2003; Leoni et al., 2001). Other advantages of NWPs include reduction in failures associated with the production process, enhanced information collection and sharing, increased diversification, and employees' satisfaction (Askenazy \& Caroli, 2010; Ramirez et al., 2007; Schøne, 2009). Hence, the increasing rate of NWPs adoption. For example, a global survey commissioned by Vodafone showed that 75 percent (three-quarters) of businesses across the globe have flexible working policies. However, while some businesses have switched from conventional methods of operating to NWPs, they do not realise they have 
done so because of a lack of knowledge of NWPs. While for the same reason, some companies interested in adopting and implementing NWPs may find it difficult. According to Helen (2016), 95 percent of employers claim to provide flexible working practices. Suppose NWPs continue to obtain regulatory approval across nations. In that case, there is a possibility of a rise in the number of NWPs that need more study to gain a better picture of the full spectrum of these activities (Kelliher \& Anderson, 2010). To ensure competitiveness and survival, technological change and development pressures demand organisations to be innovative in introducing new ways of thinking and working.

The impact of changing business practices on corporate real estate requirements has been the subject of discussion in past research (Gibson \& Lizieri, 2001; Manning \& Roulac, 2001), emphasising the office sector (Lizieri, 2003). In the management science and business organisation literature, drivers such as globalisation, innovation and convergence in IT, reorganisation and the drive for flexibility in workplaces have brought about new ways of conducting business activities. These, in turn, have altered the pattern of demand for real estate, change the locational requirements of firms and how they manage space (Lizieri, 2003). The introduction of some NWPs such as flexible working, home working, and hot-desking, implies a division of corporate space into core space, required on a permanent or longterm basis, and peripheral space which is taken on when business needs demand but is shed when surplus to requirements. A comparative study on common real-estate-related business policies and activities in Australia, Hong Kong, the UK, and the US by Gibler et al. (2002) revealed that few companies are undertaking desk-sharing, hoteling, or other property-related space intensification activities. Nonetheless, Gibson and Louargand (2001) found that NWPs have changed the nature of occupier requirements and placed greater stress on the need for flexibility in provision to allow corporate real estate strategists to manage business risk. Empirical research into NWPs and how they influence property portfolio suggests that change is muted and gradual, reflecting resistance to adopting working practices in part. Research in this area, as in many other areas of real estate, is hampered by a lack of attention to theory (Lizieri, 2003).

While NWPs is not a new paradigm, it is gaining traction in practice worldwide and yielding extensive research from various perspectives. As a result, NWPs is a progressively challenging topic to review. Although there are several relevant studies in past years, there is no comprehensive scientometric review about NWPs in the literature. Given the technological advancement which has culminated in the evolution of this field, coupled with a shortage of scientometric review, this study aims to reveal the intellectual landscape of the study of NWPs by identifying landmark research studies/articles and emerging trends to reveal the research trends in this field. Unlike the past conventional literature review study on NWPs, which are classically performed by an experienced researcher (Blok et al., 2011), this review study adopts an algorithmic method embedded in CiteSpace software. This study has advantages over previous review studies in some aspects. First, extensively published articles and a wider diverse range of NWPs-related topics are extracted. Previous review studies largely depend on keywords and are characterised by small samples. However, other text fields (e.g. titles and abstracts) can help in extracting representative terms and references to provide wider information (Chen et al., 2010). Thus, this study presents a comprehensive overview of NWPs, based on broader topic extraction that incorporates keywords, titles, and abstracts in the Web of Science (WoS) database. Second, past literature review studies mainly depend on expert opinion, but experts may not appropriately capture the emerging trends in a field (Kim \& Chen, 2015). The principle of the scientometric approach utilised in this study is to obtain a wide-ranging landscape on NWPs. The remainder of the paper is arranged as follows. It begins with the background of NWPs, followed by a discussion on the methodology employed in the study. The following section presents the analysis and discussion of results, whilst the last section concludes and suggests future research direction.

\subsection{METHODOLOGY}

For a literature review study to be a stand-alone work, a type that transcends a mere report of references (Zhou et al., 2015), a systematic approach identifies, retrieves, selects, and evaluates relevant quality publications regarding a research topic is imperative. A systematic review approach methodology was adopted in this study to elicit existing literature on NWPs to explore useful findings and identify knowledge gaps for future research directions. The process of identifying, screening, and selecting eligible research publications for this study followed a combined structured approach for scientometric review using WoS and CiteSpace software.

\subsection{Identification and Selection of Academic Journal Papers}

In effect, the scientometric analysis is established to collect literature data (Jin et al., 2017). To identify and retrieve academic journals that have published relevant papers related to NWPs from 1980 to 2018, a robust search engine, WoS database by using a topic search, was employed in this study. Aside from being a major archive for most research output, the choice of WoS rests on its ability to perform better in accuracy than other search engines. For instance, WoS offers the most reliable download function (Chen et al., 2008) and gives more accurate results in terms of centralisation of collected data (Jin et al., 2017). With the help of the WoS database, a search strategy for NWPs was developed in early November 2018 with previously identified pertinent terms relating to NWPs, following a preliminary literature review. To guarantee the use of publications of acceptable quality, only academic articles that appeared in peer-reviewed journals were included ( $\mathrm{Ng}, 2016)$. The search was limited to articles published in English-language from 1980 up till 2018 and articles not related to the topic as deemed by the authors were removed. Having conducted a systematic search on WoS to locate published articles associated with NWPs, 850 journal papers were generated for the analysis. These papers were downloaded with a full record and exported in plain text format compatible with CiteSpace. Since this is the first-ever scientometric review NWPs, the authors narrowed their search to interchangeable keywords. The search code generated by $\mathrm{WoS}$ is as follows: 
Topic: ("New working Practices" OR "new working patterns" OR "new organisational practices" OR "new ways of working" OR "workplace innovations" OR "high-performance work organisations" OR "flexible working" OR "post-Fordist work practices" OR "work transformation") Refined by DOCUMENT TYPES: (ARTICLE) AND LANGUAGES: (ENGLISH) Timespan: 1980-2018. Indexes: SCI-EXPANDED, SSCI, A\&HCI, CPCI-S, CPCI-SSH, ESCI.

\subsection{Method of Analysis}

One of the important ways by which researchers can influence industrial practice is through research publications (Cohen et al., 2002). It is believed that the number of academic publications emanating from a geographic location could determine the extent of the practice of the subject matter within the industrial sectors of such location or country (Hong et al., 2012). Osterman (1994) revealed that restructuring processes such as NWPs are widespread regarding countries and sectors, but NWPs vary across countries (Ollo-López et al., 2011). Hence, to understand the streams of NWPs-related research in different regions, the authors identified various NWPs clusters in the literature and evaluated the numbers of publications attributed to different countries, authors, institutions, and funding bodies. This study primarily employed CiteSpace to detect, analyse and visualise evolving trends and citation patterns in the literature (Chen, 2006). CiteSpace is a java application that combines information visualisation, bibliometric processes, and data mining algorithms in an interactive approach. CiteSpace portrays a simple dynamics model that enables scholarly communication in a way that a transient body of scientific publications, commonly recognised as a research front, refers to a cluster of publications in the literature, which is known as the intellectual basis (Chen, 2006).

CiteSpace enables easy identification of some special classes of articles in terms of landmarks (by eliciting citation popularity), hotspots based on a rapid increase in numbers of citations received, and pivotal papers that are essential due to their strategic position in cocitation networks (Chen et al., 2008). The method of analysis and visualisation of the identified articles in this study follows the general procedure for using CiteSpace, including time slicing, thresholding, modelling, pruning, merging, and mapping (Chen, 2004). Subsequently, the network is grouped into clusters in which each group is signifying a discrete field or a thematic focus. The larger the members of a cluster, the larger the size of the cluster, and that reflects the degree of hotspots (Jin et al., 2017). Clusters were used to determine the emerging trends in the NWPs field based on the recency of the average publication year. Also, the burst detection algorithm was applied to reveal unusually huge changes in the frequency of an entity (e.g. keywords and document citations) over time (Pollack \& Adler, 2015). CiteSpace will expose the subject field's research context and growth direction by combining the subject research frontier of network data analysis, the topic evolution trend, and the relationship between investigating the knowledge base. In comparison to the classical literature review, the CiteSpace technique can make the research context in this field more straightforward and more intuitive while still being repeatable to some extent, to eliminate the potential deviation induced by subjective judgment. Conventional narrativebased document reviews are qualitative, relying heavily on individual judgments and explanations, whereas CiteSpace software can visualise this non-descriptive judgment.

\subsection{ANALYSIS AND DISCUSSION OF RESULTS}

This review study explores various NWPs and provides insights into NWPs related research trends across the world by reviewing selected relevant research publications during the study period. The results are presented in terms of different labels associated with NWPs, a yearly frequency of publication, and research contributions by various sources. The use of scientometrics in this paper is more than a trivial measure of quantities, but rather an analysis, understanding, and ability to make managerial decisions (Klochkov, 2019). Scientometric analysis is used in determining the scholarly potential of an individual scholar, a research team, an institution, and even an entire country with varying degrees of accuracy. The use of scientometric analysis is growing because of its cheapness and faster speed of verification and the absence of a subjective "human factor" in the rapid evaluation of the publication activity of scholars. The outcome of this paper also benefits from the threshold constraints on scientometric metrics, which are a form of filter that enable unsuitable applicants to be screened out (for example, when obtaining grants). This will thereby decrease the expense of performing costly and time-consuming expert evaluations of the consistency of scholarly performance. Cocitation and cluster analyses were conducted to: (1) show the temporal evolution of NWPs, (2) define main disciplines and identify possible relationships between disciplines in the study of NWPs, (3) to evaluate widely quoted scholars and their research focus, and to investigate the major fields of study and scholarly groups that use NWPs.

\subsection{Annual Publications on NWPs Research}

Figure 1 shows the yearly publication of the analysed NWPs related studies. It revealed that only 7 papers were published in the 1980 s. This could be attributed to NWPs becoming prominent in the late 1980s and early 1990s (Bell \& Anderson, 1999). It is therefore sufficed to say that NWPs were emerging in the 1980s. Hence, it attracted only a few publications in that period. Meanwhile, the remaining identified papers were published between 1990 and 2018, indicating the period with increased research attention on NWPs. As earlier stated, technology is one of the major drivers of NWPs, and significant advancement in information technology actually took place during the 1990s (Oliner \& Sichel, 2000). The findings reveal that NWPs have become an attractive research dome for many researchers in the past three decades, and the trend may continue as long as technological innovations continue to boom. 


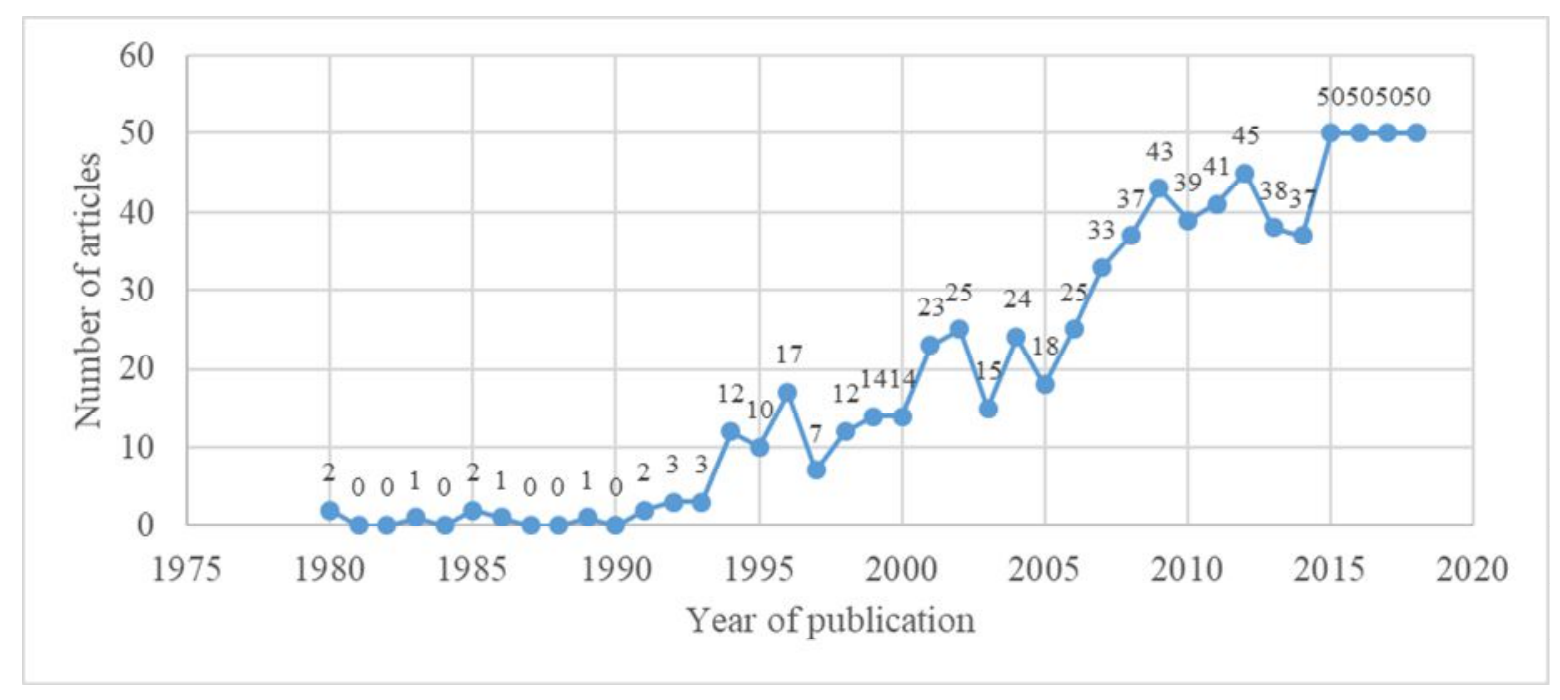

Figure 1 Annual number of publications on NWPs from 1980 to 2018

\subsection{Research Output by Authors}

Figure 2 shows the co-authorship network, representing the network of authors reflected in nodes and links, where the node denotes an identified author. Each link signifies an array of the relationship between authors being connected by the link (Zhao, 2017). To prune the network, pathfinder utility in Citespace was utilised for pruning the network. The resultant network produced 55 nodes and 40 links. The size of a node indicates the number of articles published by the corresponding author, whereas the thickness of each link signifies the intensities of collaboration between the connecting authors (Olawumi \& Chan, 2018). Four most productive authors published more than 2 articles concerning NWPs. They are; Friedhelm Nachreiner (5 articles) being a top-ranked author, Peter Vink (4 articles), Liesbeth Groenesteijn (4 articles) and Jane Lewis (4 articles), whereas other authors published 1 to 2 articles. These authors, with the exception of Lewis, have a centrality score of 0.01 each. This score quantifies the number of times a node (author in this case) acts as a bridge along the shortest path between two other nodes (authors). The betweenness centrality is used to quantify the control of a human on the communication between other humans in a social network (Freeman, 1977). Hence, these authors with betweenness centrality have an influence to some extent over other authors in the scientific network.

Considering the established collaborative efforts among the authors in this research domain, the co-authorship network portrayed numerous research communities comprising some productive focal authors cooperating with other authors in the network. Two principal research communities were identified: (1) community constituted by the most productive author, Nachreiner F., as the central author, collaborating with other authors such as Janssen, D., Giebel O., and Wirtz A. Friedhelm Nachreiner is a professor at Carl von Ossietzky University of Oldenburg, Germany and his research focus is on shift work, mental workload, and monitoring. As the most productive author in terms of publications, he has collaborated with other researchers in this domain; (2) robust collaboration in the research circuit of Groenesteijn L. and Vink P. as the central authors, forming a community with other researchers such as Blok M., Formanoy M., De Korte E. and Schelris R. This research community focuses on New Ways of Working (NWW) and flexibility in workplaces. 
PEDERSEN VB

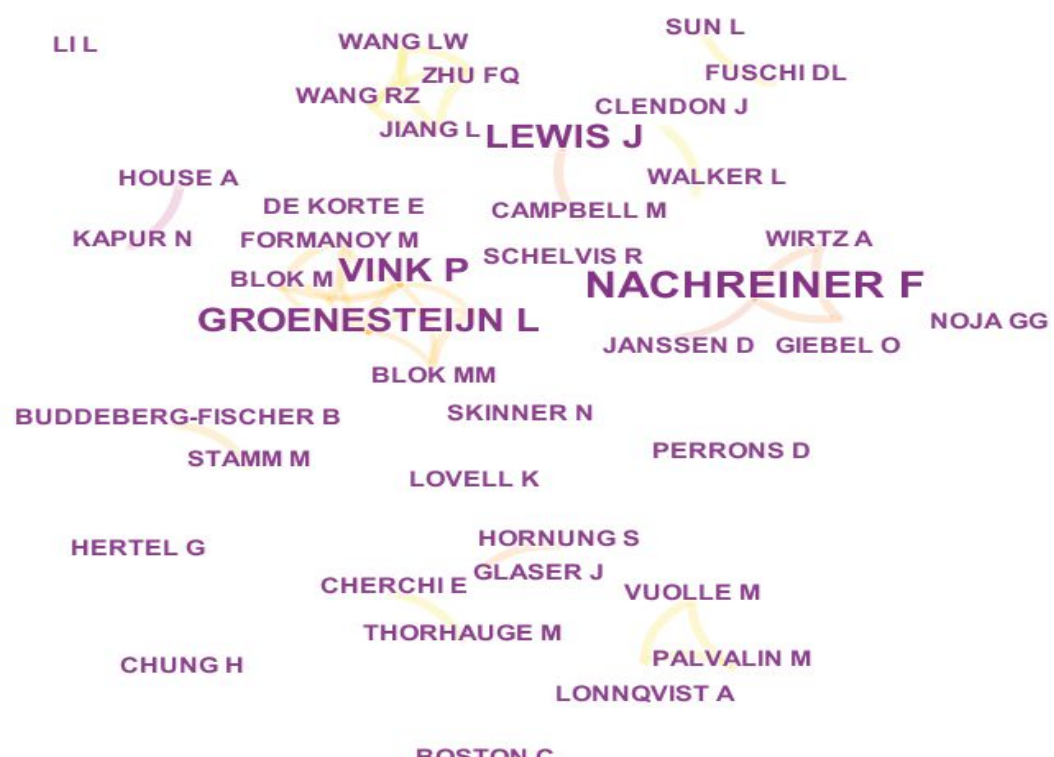

Figure 2 Co-authorship network

\subsection{Research Output by Countries}

The number of publications emanating from a country in a research area is a good yardstick to measure how policies regarding such research are being practiced in that country. As illustrated in Table 1, during the studied period, NWPs related studies have been conducted mostly in developed countries. This shows that the contribution of developed countries towards NWPs research is very significant. The predominance of NWPs research in developed nations indicates that NWPs research is not yet a global interest. This is logically meaningful because of the great emphasis on flexible working practices in the developed world. The numerous flexible working initiatives can also explain this by developed nations in enhancing the adoption of NWPs. For instance, most governments are currently promulgating some useful legislation and policies supporting NWPs (such as equal benefits for both part-time and full-time employees). The high betweenness centrality scores as identified in Table 1 also indicate the significant influence of these countries on the NWPs research field.

Table 1 shows the 10 top-ranked countries by citation counts (in descending order). The results revealed that the UK (England and Scotland) is the leading country with the highest number of publications, which is $41.61 \%$, confirming the prevalence of NWPs in the region. A previous study in the UK (Millward, 1992) revealed that the spread of NWPs in the region resulted from the removal of barriers to NWPs by firms' managers who are responding to changes in the technological environment rapid globalisation in the business world. European countries including Finland, the Netherlands, Germany, and Sweden, recorded 123 articles and ranked the second-highest number of publications on NWPs related research. This result affirms the recent finding in the literature that NWPs have significantly increased across firms in European countries (Goñi-Legaz \& Ollo-López, 2015). Although NWPs originated from the US (Arabandi, 2011), contrary to Eriksson (2003) who observed that NWPs are common only in the US because of scanty evidence about the spread of NWPs in other parts of the world, it is clear that NWPs have now prevalently extended beyond its origin as UK and Europe are now leading the US in terms of numbers of publications. The results show that China is the leading country in Asia ( 30 publications) followed by South Korea with 6 publications. Other Asian countries that made the list include Japan, Thailand, and India, with 2 publications each. Aside from South Korea, other Asian tigers (Hong Kong, Singapore, and Taiwan) were not included in the list, indicating research potential in this area in those countries. It was found that 4 publications come from Africa as a continent ( 2 publications each from Ghana and South Africa). Various factors could be attributed to the lack of research output in Africa, including an immature sense of publication, NWPs are yet to be embraced as a priority in business workplaces, and lack of understanding of the potential benefits of NWPs. Hence, there is great potential for NWPs research in African countries. 
Table 1 Publication frequency by countries

\begin{tabular}{lllll}
\hline Frequency & Centrality scores & Country & $\%$ & Rank \\
\hline 287 & 0.56 & ENGLAND & 37.91 & 1 \\
92 & 0.38 & USA & 12.15 & 2 \\
73 & 0.11 & AUSTRALIA & 9.64 & 3 \\
41 & 0.24 & NETHERLANDS & 5.42 & 4 \\
36 & 0.03 & GERMANY & 4.76 & 5 \\
30 & 0.03 & PEOPLES R CHINA & 3.96 & 6 \\
28 & 0.12 & SCOTLAND & 3.70 & 7 \\
24 & 0 & FINLAND & 3.17 & 8 \\
22 & 0.05 & SWEDEN & 2.91 & 9 \\
16 & 0.22 & CANADA & 2.11 & 10 \\
\hline
\end{tabular}

Furthermore, citation bursts are another important tool to understand research areas and domains. Citation bursts indicate a strong surge of frequency abrupt change in citation counts in a given period (Chen et al., 2014). Significant citation bursts were recorded in 6 countries (see Figure 3). All the nodes that exhibited citation bursts represent countries or prominent regions with significant studies and research in NWPs. It was noted that the USA, England Netherland, and Finland, recorded high frequencies and high citation bursts. Moreover, 4 out of the 6 countries with citation burst are European nations, indicating that there are significant research effort and devotion to NWPs in Europe. Moreover, the most recent citation bursts are connected to European countries.

\section{Top 6 Countries with the Strongest Citation Bursts}

\begin{tabular}{llrrr}
\multicolumn{1}{c}{ Countries } & \multicolumn{2}{c}{ Year Strength Begin End } \\
USA & 1980 & 5.1548 & $\mathbf{2 0 0 0}$ & 2004 \\
ENGLAND & 1980 & 4.4165 & $\mathbf{2 0 0 5}$ & 2007 \\
DENMARK & 1980 & 3.7905 & $\mathbf{2 0 1 1}$ & 2012 \\
NETHERLANDS & 1980 & 4.5945 & $\mathbf{2 0 1 2}$ & 2016 \\
FRANCE & 1980 & 4.375 & $\mathbf{2 0 1 4}$ & 2015 \\
FINLAND & 1980 & 4.0976 & $\mathbf{2 0 1 5}$ & 2018
\end{tabular}

Figure 3 Country citation burst

\subsection{Author Co-Citation Analysis}

With the aid of a dataset collected from WoS, CiteSpace was employed to show visualisation and analysis of how authors are co-cited within the research area of NWPs from 1980 to 2018. As shown in Figure 4, there are 687 nodes and 2201 links with pathfinder pruning, forming a merged author cocitation network with 17 clusters (red labels) obtained and labelled by locally linear regression (LLR). In CiteSpace, terms are selected for clustering by three different algorithms, LSA, LLR, and mutual information. LLR was chosen for this analysis because it reflects the unique aspect of a cluster (Chen et al., 2010). The purple labels represent nodes that stand for authors and institutions with the sizes corresponding to the number of times they were cited within the study period. Each node represents an author or organisation, and the links connecting the nodes represent direct partnerships established through the co-authorship of papers. The network is divided into 17 cocitation clusters. Index terms from their own citers label these clusters. Five major clusters have been generated with more than 50 members and named with, mediating role $(106,2008)$, labour market transformation $(77,2004)$, individual performance $(76$, 2013), schedule flexibility (73, 2012), and activity-based flexible office $(62,2015)$ by CiteSpace based on LLR algorithm. Note, corresponding citation size and mean citation year for each cluster are enclosed in parentheses. The largest cluster (mediating role) has 106 members and the mean citation year of 2008. The most active citer to this cluster is Atkinson and Hall (2011), who explore the influence of flexible working on employee happiness and attitudes. 


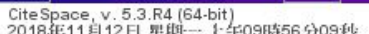

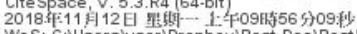

WoS: C:IUsers WuseriDropboxIPost DoclPostDoc Dr WadulPapersIWWP LRICiteSpaceWof SIDownload

Timespan: 1980.2018 (Slice Length $=1$ )
Selection Criteria: Top 50 per slice, LRF 2 , LBY $=8, e=2.0$

Network: $N=687, E=1856$ (Density $=0.0079$ )

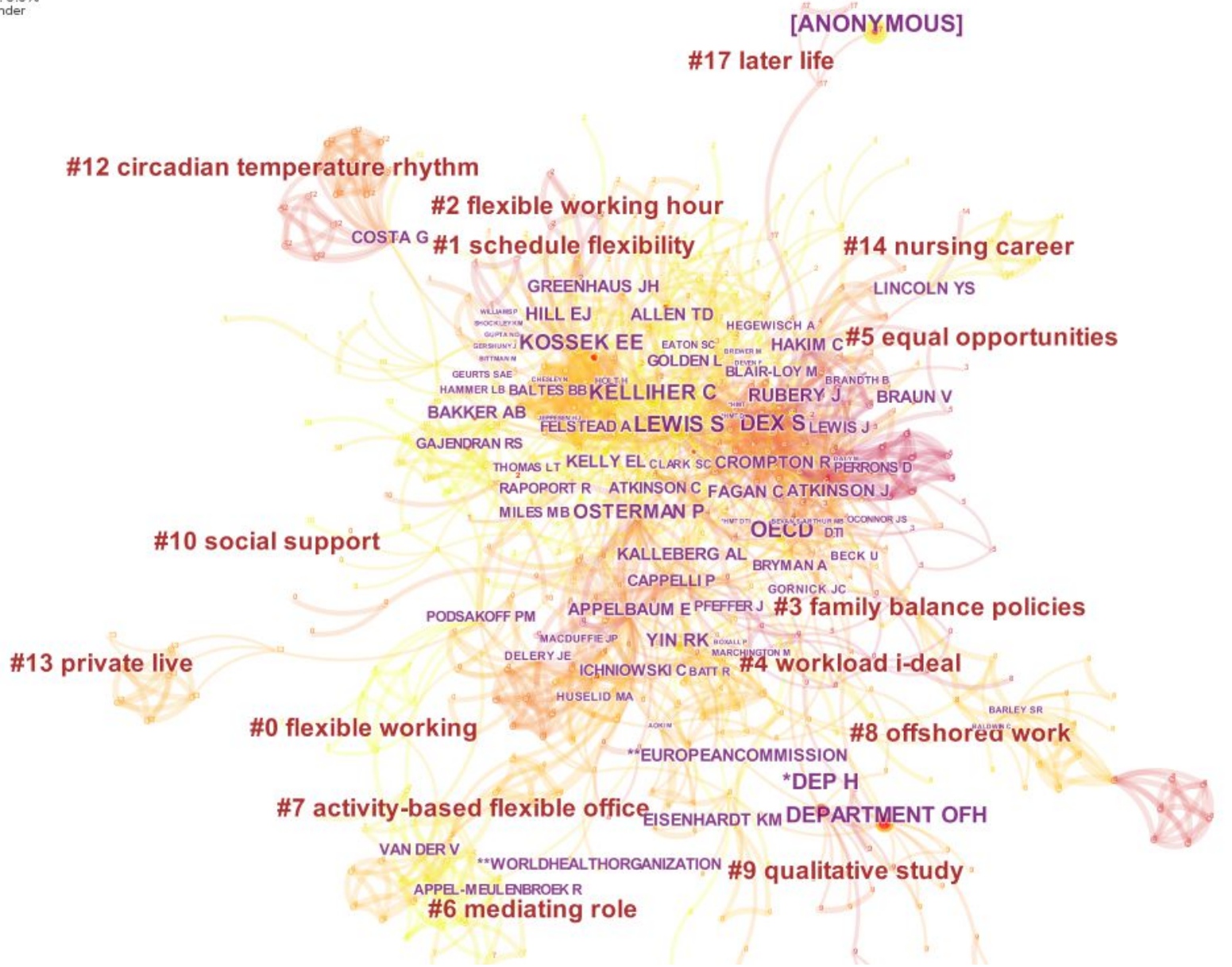

Figure 4 Merged author cocitation network with labels for clusters

Table 2 presents detailed information about the top ten most-cited authors or organisations with the authors belonging to different clusters It was observed that the same organisation appeared in different names in different articles and is therefore identified differently by CiteSpace. The results show that the Department of Health (DH) can equally be identified by *dep H and Department of $\mathrm{H}$ (both from cluster 6). Hence, the most cited author is DH, the branch of the United Kingdom government concerned with the maintenance of public health. DH is responsible for providing leadership for the National Health Service (NHS) and the government's social care and public health agendas. In collaboration with the National Institute of Health Research (NIHR), DH also provides funding support for health research, including NWPs related studies (for example, Smith \& Ward, 2015). The next top-ranked author by citation counts is [Anonymous] in Cluster \#5, with citation counts of 66. Anonymous authorship is unreliable because it might combine many references without precise author information (Gong et al., 2013). Therefore, the actual second most cited author is Lewis Susan in Cluster \#3, with citation counts of 59 and the mean citation year of 2002. Lewis Susan is a Professor at the Business School of the Middlesex University, London, United Kingdom. Her research focuses on gender and 'work-life balance' issues and workplace practice, culture and change, flexible working arrangements, all in diverse national contexts. Shirley Dex, in Cluster \#1, having 43 citation counts, is the third most cited author. Shirley Dex is an Emeritus Professor of Longitudinal Social Research at UCL Institute of Education, University College London. Interestingly, most of the top-cited authors and organisations are based in the UK, indicating that NWPs related research is famous in European countries. 
Table 2 The top ten most-cited authors

\begin{tabular}{lllll}
\hline Citation counts & References & Cluster \# & Country & Institution \\
\hline 59 & Lewis S. & 3 & UK & Middlesex University, London \\
43 & Dex S & 1 & UK & University College London \\
35 & Kossek EE & 3 & USA & Purdue University \\
35 & Kelliher C & 5 & UK & Cranfield University \\
28 & Rubery J & 2 & UK & University of Manchester \\
28 & Osterman P & 0 & USA & MIT Sloan School of Management, Cambridge \\
\hline
\end{tabular}

The changes happening among productive authors in a field can be described in part by authors that have gained an abrupt increase of citations, which refer to citation bursts. Figure 5 is a visualisation of the burst analysis of the cited authors in the dataset from 1980 to 2018, showing the top 21 bursting authors, as sorted by the burst weight. It can be seen in Figure 5 that the burst in author citation continues from 1995 to 2018. The earliest burst is attributed to Handy C with his prominent publication on 'trust and the virtual organisation' (Handy, 1995) being cited 416 times from the Web of Science Core Collection as at the time of this study. The author with the overall strongest burst is the Department of Health based in the UK, as discussed earlier. Although the author was identified as two different entities by CiteSpace that is *Dep H and Department H, with burst strengths of 15.69 and 10.91, respectively, they belong to the same citation burst as their bursts both begin in 2002 and end in 2010. This implies that the Department of Health significantly impacted the NWPs research community between 2002 and 2010. The author with the most recent strongest citation is Allen Tammy of the University of South Florida, United States, with a burst strength of 4.77 beginning from 2016 and still bursting. Her publication with the highest citations (Allen et al., 2000) discussed issues concerning the measurement of work-family conflict.

\section{Top 21 Cited Authors with the Strongest Citation Bursts}

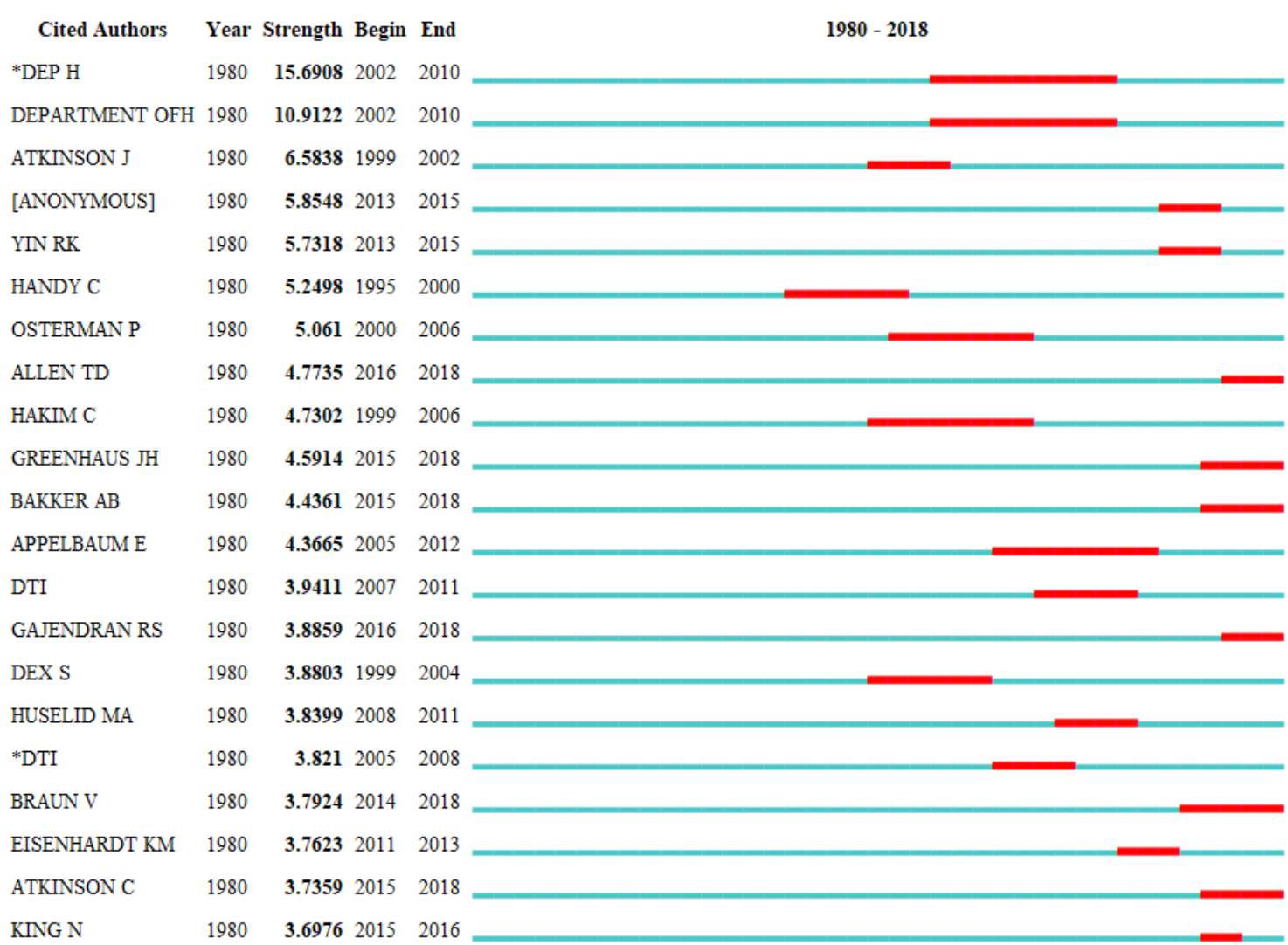

Figure 5 Top 21 authors with the strongest citation bursts

\subsection{Document Co-Citation Analysis}

Figure 6 shows a timeline visualisation of cocitation references from 1980 to 2018, containing 334 nodes and 603 links. The nodes in the figure symbolise cited references, while the links denote the cocitation relationship. The colour pattern at the topmost part of the Figure follows a gradual change (in line with time slices across the study period) from blue (1980) to yellow (2018). The colours of cocitation links are set to reveal time slice that a connection was made for the first time. The colour of a node represents the time slice that a reference 
belongs to, while cluster colour shows the time slice for the average year of a cluster member references' publication date (Chen et al., 2014). The red shaded circle represents certain time slices. The citation frequency of a corresponding paper abruptly increased, signifying citation burst, and the circle's size reflects how strong the burst is. References that have high betweenness centrality are highlighted nodes with a purple ring in the network, pointing to pivotal nodes that connect two clusters.

Cluster symbolises a concentration of themes in the bibliographic settings. CiteSpace's cluster recognition trajectory is used to simulate the development trends of NWPs research. To establish the nature of a cluster of co-cited references in a network, CiteSpace algorithmically generates cluster labels, identifies symbolic concepts in the cluster, and provides rich text from an interesting article (Chen, 2013). The cluster labels are obtained from a pool of references. The reference clusters of NWPs are labelled in ascending order based on the cluster size. Thus, individual performance, longitudinal effect, and contagious flexibility are the three largest clusters in the network, revealing the major research hotspots of NWPs.

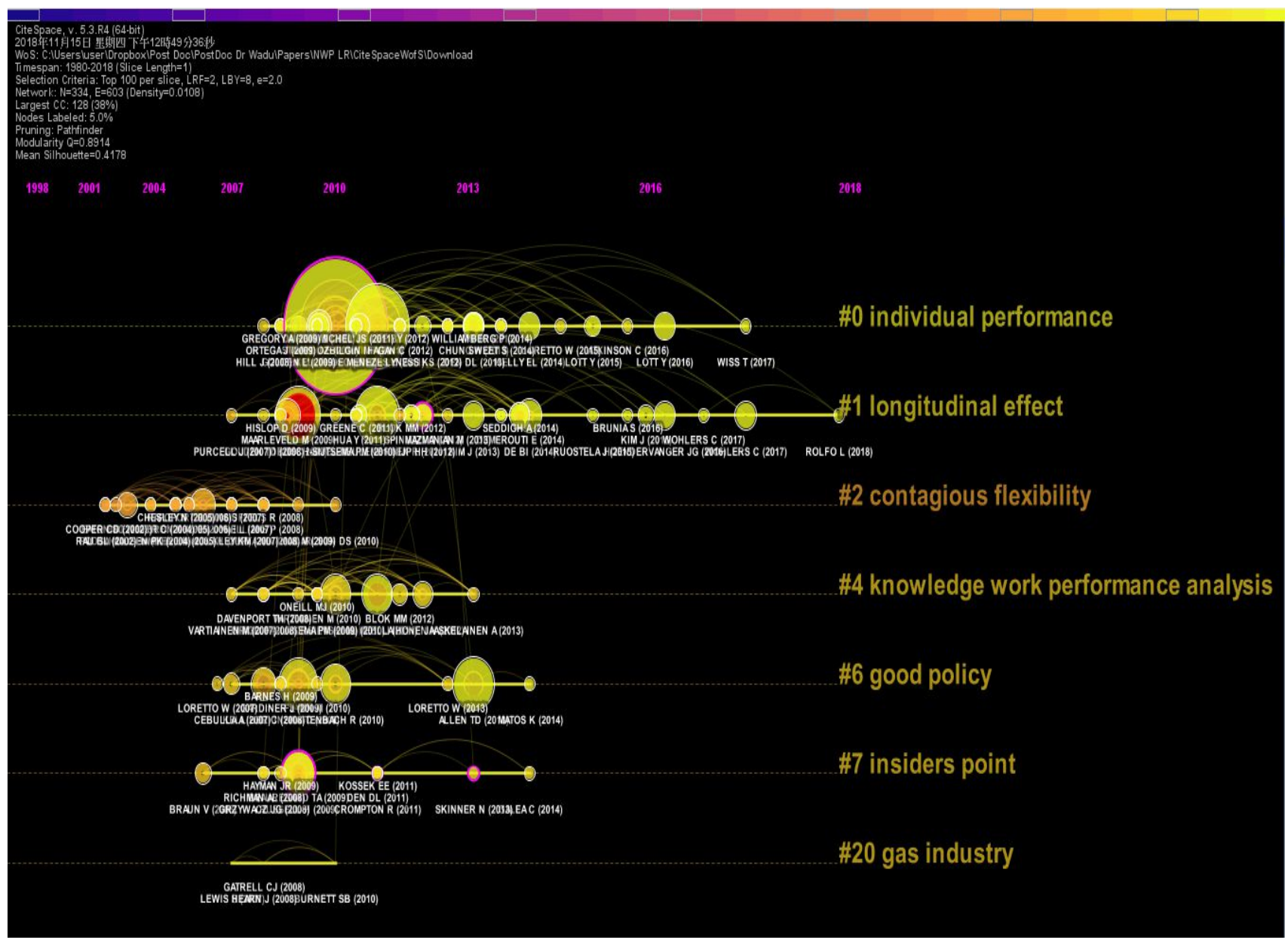

Figure 6 Timeline view of cocitation references

The network is divided into 7 cocitation clusters. Index terms from their own citers label these clusters. The largest 2 clusters were automatically summarised by CiteSpace and were named by LLR. The largest cluster (numbered as \#0) has 37 members and was labelled as an individual performance by LLR. The most active citer in this cluster is Chung (2018). The study by the active citer used data from 30 European countries to examine workers' access to family-friendly working-time arrangements (WTA) including flexitime, and time off work for personal reasons. The second largest cluster (numbered as \#1) has 31 members and was labelled as the longitudinal effect by LLR. Moreover, the most active citer to cluster \#1 is Wohlers and Hertel (2018), which analysed the longitudinal effects of activity-based flexible office design on teamwork. It was found that cluster label by LLR is influenced by the title of the most citer paper to the cluster. The average year of publication of cluster \#0 (individual performance) and cluster \#1 (longitudinal effect) is 2012, which shows that the longitudinal impact of individual performance in an environment where flexible working arrangements are practised is still the emergent trend NWPs field.

The landmark in any knowledge field can be identified by studies that attract a high number of citations (Chen et al., 2010). CiteSpace generated the top 10 widely cited papers of landmark importance and ranked according to citation counts. The top-ranked article by citation counts is Kelliher and Anderson (2010) in Cluster \#0, with citation counts of 21. The second-ranked article, with citation counts of 13, is de Menezes and Kelliher (2011) also in Cluster \#0. Two references (Appel-Meulenbroek et al., 2011; Yin, 2009) were ranked third because they have the same citation counts of 9, and they are from the same Cluster \#1. The first reference (Kelliher \& Anderson, 2010) 
entitled "Doing more with less? Flexible working practices and the intensification of work", is an empirical study that examines an unanticipated consequence of adopting flexible working practices. The study revealed that flexible workers are more satisfied and committed to their jobs than their non-flexible counterparts. In the second reference (de Menezes \& Kelliher, 2017), the authors researched the relationship between flexible working arrangements and individual employee performance, demonstrating that flexible working arrangements have positive indirect effects on worker performance. One of the two references that ranked third (Appel-Meulenbroek et al., 2011) underlined some known benefits and disadvantages of the activity-based office concept. Whereas the second one (Yin, 2009) of the same ranking provided an essential guide to qualitative methods in organisational research with an extensive emphasis on the use of a case study approach. In all, the top ten landmark articles revealed that references that have a high impact in the research community began to surge after 2008 with the 4 articles published in 2009 highlighting the earlier research hotspots about NWPs.

Moreover, the highlighted nodes with a purple ring, as shown in Figure 6, are the references with high betweenness centrality in the network. These are central articles that connect two clusters in the network. The top 4 ranked references by centrality with equal centrality of 0.13 are: Kelliher and Anderson (2010); Nijp et al. (2012); Kossek et al. (2011) and Skinner and Chapman (2013) in Cluster \#0, in Cluster \#1, \#7, and \#7, respectively. The only reference with an abrupt burst citation pattern is the article by Huselid (1995), which comprehensively assessed the relationships between systems of high-performance work practices (HPWPs) and firm performance. The results showed that HPWPs have an economically and statistically significant impact on employee turnover and productivity on the one hand and corporate financial performance on the other hand.

\subsection{Keywords Co-Citation Analysis}

Keywords provide an understanding of the basic content of articles while a keyword network analysis enables one to scrutinise research topics and establish the evolving frontiers of knowledge (Lee \& Su, 2010). Figure 7 shows the co-occurring keywords network of NWPs, containing 271 nodes and 956 links from 1980 to 2018, with each node denoting a distinct keyword and a link between two nodes are corelationship between the connecting keywords. In total, 271 keywords extracted are organised together with the clusters in the network as shown in Figure 7. Cluster analysis can further help to identify the evolution of NWPs research. The network is divided into 10 cocitation clusters. Index terms from their own citers label these clusters. Clusters are numbered in such a way that higher ranks are issued to clusters with more references. The largest cluster $(\# 0)$ has 49 members and was labelled as flexible workplace practice by LLR. The second-largest cluster (\#1) has 32 members and was labelled as other stakeholders by LLR. The third largest cluster (\#2) has 32 members and was labelled as the activity-based flexible office by LLR. As the keywords in the ten clusters were distinct, it was decided that these were representative of the different NWPs trends. Considering both the size and recency of member nodes, clusters $\# 0$ through $\# 2$ are representing emerging research themes in the domain. The references in this cluster influenced successive research in NWPs studies. 


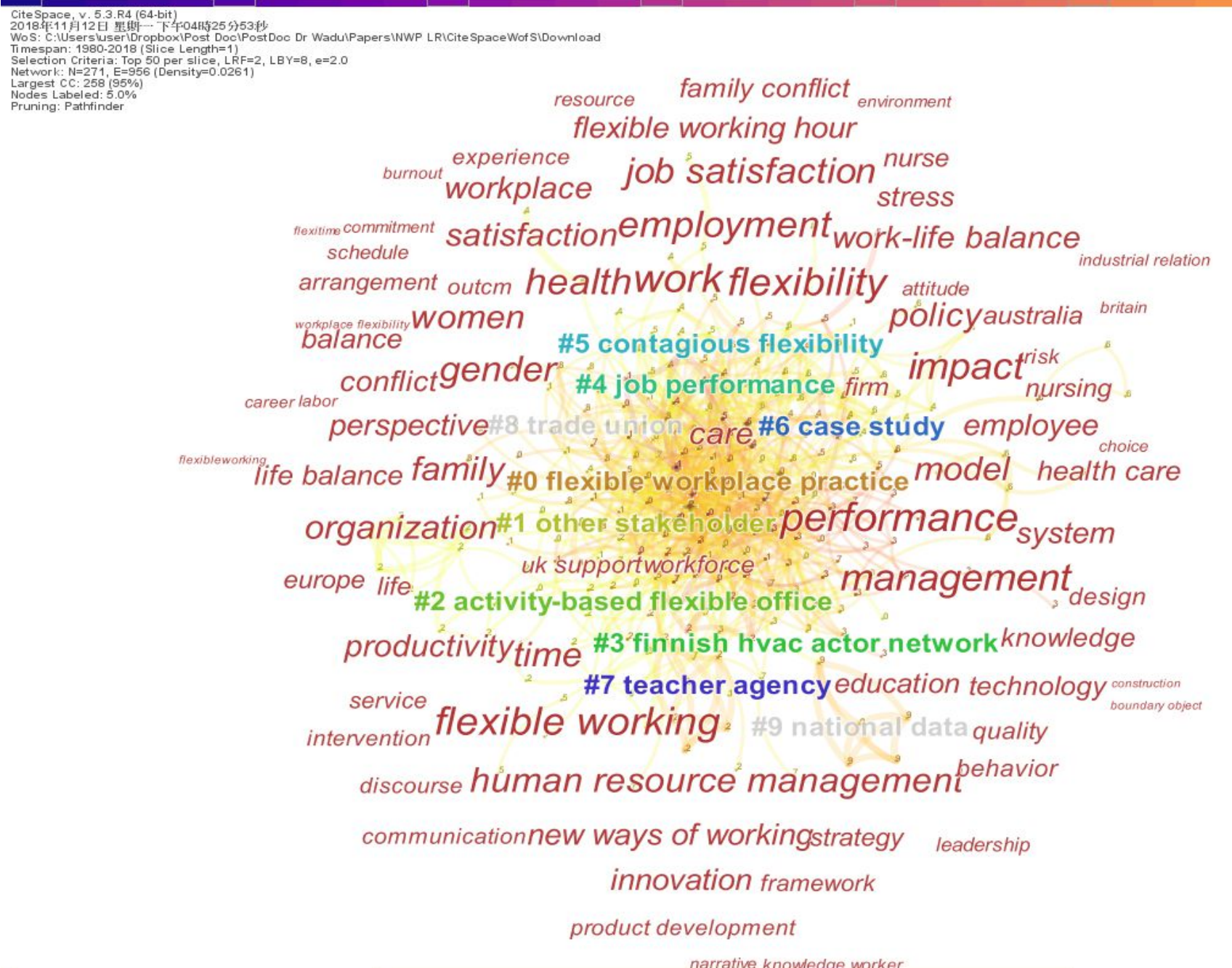

Figure 7 Merged network of co-occurring keywords and clusters

The frequency of keyword citations can reveal the emerging research hotspots of a research domain, while the associated mean publication year reflects the trend of those hotspots (Chen et al., 2006). Based on the frequency of keyword, the top-ranked keywords by citation counts (note: the years in parenthesis denote mean cite years) is performance (2004) with citation counts of 60, while work (1996) occupied the second position with citation counts of 58, flexible working (1998) is third, with citation counts of 51, the fourth one is flexibility which could be another name for flexible working. Meanwhile, these two entities were not merged to appear as a single node because flexibility is a broad word that could have different meanings, such as flexible office arrangement or work arrangement. In essence, the top-ranked keywords (i.e. performance, work, and flexible working) are hotspots in NWPs research. One important hotspot (health) reflects a different application field of NWPs.

Although the mean cite years may suggest the evolution of top hotspots to some extent, the overall understanding of the period that each keyword occurred is not clear enough (Jin et al., 2017). Therefore, Figure 8, provides a clear visualisation of the overall emerging trends by showing the time zone of co-occurring keyword network. The network's time zone enables chronological visualisation of the most applied keywords, revealing the structure of the trend of research hotspots on NWPs at varying times. However, this further presentation does not change the meanings of relevant parameters as displayed in Figure 7. As shown in Figure 8, NWPs research has concentrated on 'work' is the first hotspot that attracted the research community's attention. The results also showed that the emerging trends of NWPs application include life balance, boundary object, and environment. The findings can enable researchers to decide the status of research topics and identify new directions for potential research. 


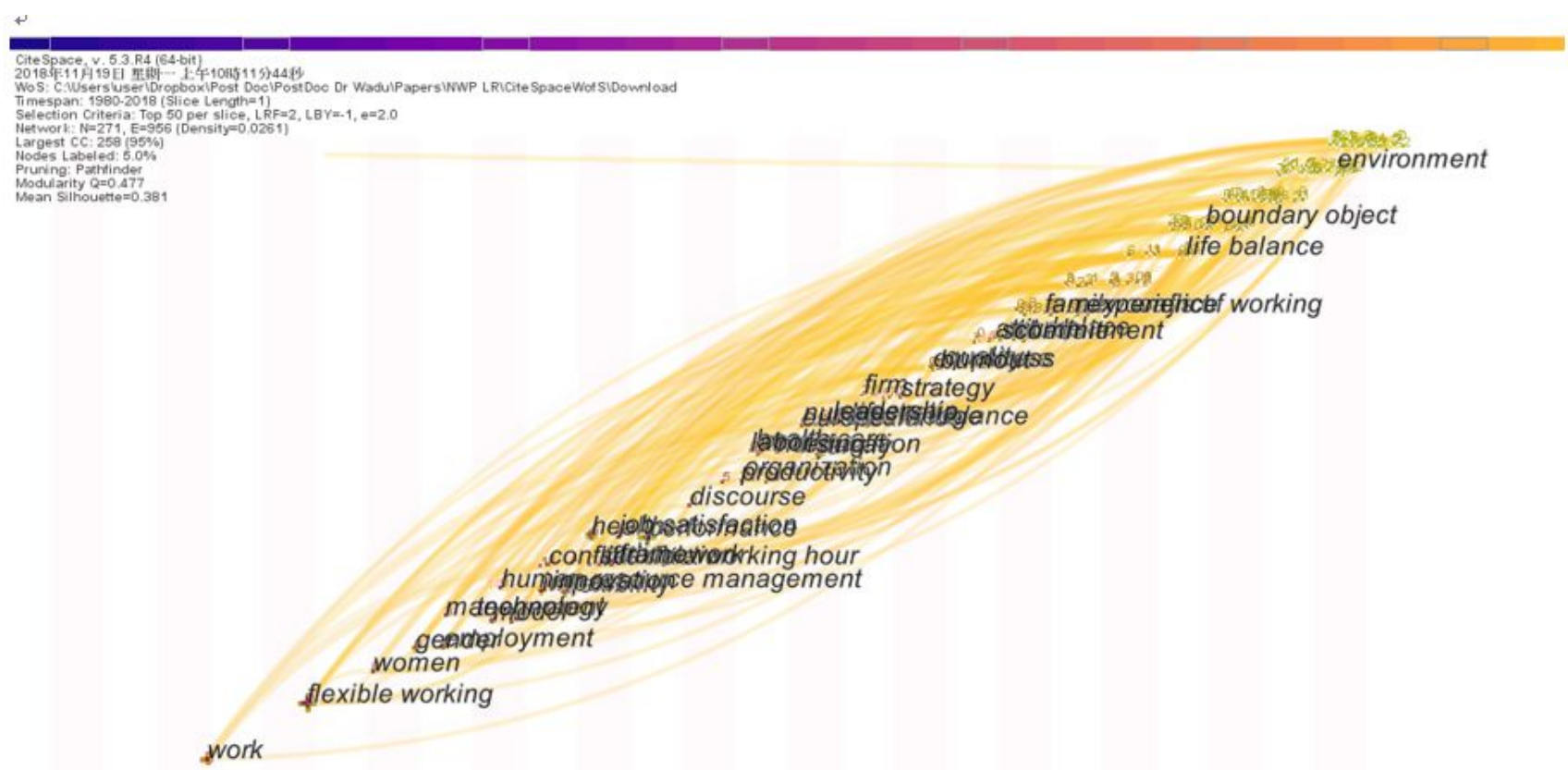

$19931994199519981997199819992000200120022003200420052006200720082009201020112012201320142015201620172018 \quad 4$

Figure 8 Timeline view of the keywords

Only 5 keywords exhibited citation bursts in their appearances between 1980 and 2018. Keywords associated with an uptick in citations, which often occurred over time, were thought to be indicators of frontier topics or new research developments. Keywords started to burst since 1996 with work being the first bursting keyword from 1996 till 2004 with a burst strength of 5.91. This is followed by impact bursting from 2002 till 2010 with a burst strength of 4.65. These two keywords have the equal longest period of a burst of 8 years, but they do not have the strongest citation strength. This implies that the research focusing on these keywords has drawn the research community's continuous attention, but a massive explosive growth was not noticed. Flexible working hour is another important keyword with a relatively long period of burst beginning from 2003 till 2010. The keyword with the strongest burst is care which is related to health and family issues, and interestingly, this keyword has the most recent burst, lasting from 2007 till 2011 . Hence, the main research frontier in terms of burst strength for NWPs research is care, revealing that care is pivotal to the development of NWPs research. On the whole, the results show that the hotspots for NWPs research significantly evolved after the 1990s.

\subsection{CONCLUSION}

Using CiteSpace to carry out scientometric analyses (including author, country, co-word, cocitation, and clusters), several research frontiers and important nodes were identified in the NWPs literature. The study revealed that there is a remarkable increase in publications from 2 papers in 1980 to 50 papers in 2018. In terms of research output by authors, it was revealed that Friedhelm Nachreiner, Peter Vink, Liesbeth Groenesteijn, and Jane Lewis are the top four lead authors in the field. However, these authors received limited citations and none of them received citation burst over the study period. Meanwhile, the topmost cited authors, including Lewis Susan, Department of Health and Shirley Dex, are all originated from the UK. Although the Department of Health recorded the overall citation burst, the author with the most recent strongest citation burst is Allen Tammy (2016 to date). The notable authors can be contacted for research collaboration or expert panelists. Prospective research students can also shortlist them for research supervision. The findings also revealed that the majority of the research output is from the UK, USA, Europe, Australia, and China. Although the USA recorded the strongest citation strength, the most recent citation bursts are associated with European countries, indicating that NWPs research is trending in Europe and is attracting the research community's attention. Regarding keywords, the largest cluster which represents a prominent research hotspot is identified as flexible working arrangements. Meanwhile, the latest research trend focuses on the environment. Kelliher and Anderson (2010) and de Menezes and Kelliher (2011) are the most cited documents, but only one document (Huselid, 1995) received citation burst. Also, the largest 2 clusters are individual performance and longitudinal effect, indicating emerging trends and hotspots in NWPs research. This information will guide interested researchers in searching for relevant materials. The main research frontier in terms of burst strength for NWPs research is care which is useful information for interested researchers willing to apply for grants in this domain.

As the workplace continues to respond to technological advancement, NWPs will also continue to advance. This calls for more distinct research works that meet the organisation's needs and cater to employees' wellbeing. This research direction can be explored 
further by interested researchers from different fields to provide more insights into identifying reasonable work practices in other firms. This is imperative because work practices differ based on nature and cultural routine in various organisations. Given that technology has been a critical enabler of NWPs trend and innovations in technology increasingly ubiquitous, NWPs are gradually becoming and may eventually become the norm. Therefore, developing countries, especially Africa, must heed this challenge by exploring valuable research potentials in NWPs. To date, research on NWPs has given special attention to specific subjects while being severely biased toward others. The performance aspect of NWPs has been a central focus, whereas studies about the impact of NWPs on real estate development are largely missing. Scholars in the NWPs area must widen their focus beyond performance and work to consider and include the changes that real estate and property development brought about by NWPs. As the topic of NWPs has become commonplace within industry and academia, it is high time for researchers to shift their focus to creating clusters of research projects that focus on particular issues relating to new ways of working. Diverse NWPs are revealed in the literature with a different approach in different countries and institutions; hence, every country should develop NWPs best suited to its specific market. As a result, future research is a huge opportunity to focus on developing and disseminating NWPs for nations that have yet to create their approach. According to the findings of this research, there is a need for more active collaboration among the actors across organisations, institutions and countries that are engaged in NWPs, research collaboration offers great benefits. Research institutions and financing agencies interested in NWPs research should develop policies to encourage global, interinstitutional, and interdisciplinary research cooperation as a prerequisite for applying for similar funding schemes.

While this paper provides useful information, users should be cautious of some limitations in the findings from this study. First, the authors only collected bibliographic records on NWPs from one database, namely WoS which selectively index publications. Hence, the possibility of partially investigating intellectual hotspots, landmark articles, and emerging trends in NWPs research cannot be ruled out. Second, the authors recognised that some important articles could be missing especially if the query phrases for topic search in WoS do not exist in the indexing terms. To address this, we employed possible variations of queries relevant to NWPs studies, hence the finding in this article is highly generalisable. However, readers should bear in mind that no single international bibliographic database considers all of the author's publications typically. Also, the literature was searched using seemingly broad keywords, thus overestimating the final number relevant to real estate research. Future research should use more precise keywords and focus on a specific field. Thirdly, only articles were used as research objects for quality information, which may omit important records. Future research can include other research objects such as books and conference proceedings. There are also limitations to using Citespace software the most significant of which is the learning curve needed to set proper visualisation parameters. Furthermore, some maps and clusters may be highly complex, necessitating specialised domain expertise for interpretation. There is a need to compare the analysis conducted by human experts to the interpretation cues provided by Citespace automatic labelling and summarisation methods. Therefore, a cross-study comparison is recommended in future research to establish a comparable platform to accommodate findings made by different researchers regarding NWPs. In all, the results of this study will allow researchers, particularly beginners, to understand the context, current status, and outcomes of NWPs as a field of study and provide a guide for future research.

\section{References}

Allen, T. D., Herst, D. E. L., Bruck, C. S., \& Sutton, M. (2000). Consequences associated with work-to-family conflict: A review and agenda for future research. Journal of Occupational Health Psychology, 5(2), 278-308.

Appel-Meulenbroek, R., Groenen, P., \& Janssen, I. (2011). An end user's perspective on activity-based office concepts. Journal of Corporate Real Estate, 13(2), 122135 .

Arabandi, B. (2011). Globalisation, Flexibility and New Workplace Culture in the United States and India. Sociology Compass, 5(7), 525-539.

Askenazy, P., \& Caroli, E. (2010). Innovative work practices, information technologies, and working conditions: Evidence for France. Industrial Relations: A Journal of Economy and Society, 49(4), 544-565.

Askenazy, P., Caroli, E., \& Marcus, V. (2002). New organizational practices and working conditions: Evidence from France in the 1990's. Recherches Économiques de Louvain, 68(1-2), 91-110.

Atkinson C and Hall L. (2009) The Role of Gender in Varying Forms of Flexible Working. Gender Work and Organisation 16: 650-666.

Atkinson, C., \& Hall, L. (2011). Flexible working and happiness in the NHS. Employee Relations, 33(2), 88-105.

Bailey, K., \& Breslin, D. (2021). The COVID-19 pandemic: What can we learn from past research in organisations and management? International Journal of Management Reviews, 23(1), 3-6.

Bell, S. M., \& Anderson M. (1999). Workplace solutions. Journal of Corporate Real Estate, 1(4), 349-360.

Blok, M., Groenesteijn, L., van den Berg, C., \& Vink, P. (2011, July). New ways of working: a proposed framework and literature review. In M. M. Robertson (Ed.), EHAWC 2011. Ergonomics and Health Aspects of Work with Computers - Lecture Notes in Computer Science (Vol. 6779, pp. 3-12). Heidelberg: Springer.

Chen, C. (2004). Searching for intellectual turning points: Progressive knowledge domain visualisation. Proceedings of the National Academy of Sciences of the United States of America, 101, 5303-5310.

Chen, C. (2006). CiteSpace II: Detecting and visualising emerging trends and transient patterns in scientific literature. Journal of the American Society for Information Science and Technology, 57(3), 359-377.

Chen, C. (2013). Hindsight, insight, and foresight: A multi-level structural variation approach to the study of a scientific field. Technology Analysis \& Strategic Management, 25(6), 619-640.

Chen, C., Dubin, R. \& Kim, M. C. (2014). Orphan drugs and rare diseases: A scientometric review (2000-2014). Expert Opinion on Orphan Drugs, 2(7), 709-724.

Chen, C., Ibekwe-SanJuan, F., SanJuan, E., \& Weaver, C. (2006, October 31-November 2). Visual analysis of conflicting opinions. In P. C. Wong \& D. Keim (Eds.), Proceedings of the IEEE Symposium on Visual Analytics Science and Technology (VAST) (pp. 59-66). Los Alamitos, CA: IEEE.

Chen, C., Ibekwe-SanJuan, F., \& Hou, J. (2010). The structure and dynamics of cocitation clusters: A multiple-perspective cocitation analysis. Journal of the American Society for Information Science and Technology, 61(7), 1386-1409.

Chen, C., Song, I.-Y., Yuan, X., \& Zhang, J. (2008). The thematic and citation landscape of Data and Knowledge Engineering (1985-2007). Data \& Knowledge Engineering 67(2), 234-259.

Chung, H. (2018). Dualization and the access to occupational family-friendly working-time arrangements across Europe. Social Policy \& Administration, 52(2), 491507.

Cohen, W. M., Nelson, R. R., \& Walsh, J. P. (2002). Links and impacts: The influence of public research on industrial R\&D. Management Science, 48(1), 1-23. 
Delanoeije, J., \& Verbruggen, M. (2019). The use of work-home practices and work-home conflict: Examining the role of volition and perceived pressure in a multimethod study. Frontiers in Psychology, 10, 2362.

de Menezes, L. M., \& Kelliher, C. (2011). Flexible working and performance: A systematic review of the evidence for a business case. International Journal of Management Reviews, 13(4), 452-474.

De Menezes, L. M., \& Kelliher, C. (2017). Flexible working, individual performance, and employee attitudes: Comparing formal and informal arrangements. Human Resource Management, 56(6), 1051-1070.

Dixon, M., \& Ross, P. (2011). VWork: Measuring the benefits of agility at work. Hampton Wick: Unwired Ventures.

Dixon, T. (2005). The impact of information and communications technology on commercial real estate in the new economy. Journal of Property Investment Finance, 23(6), 480-493.

Eriksson, T. (2003). The effects of new work practices: Evidence from employer-employee data. In T. Kato \& J. Pliskin (Eds.), Advances in the economic analysis of participatory \& labor-managed firms (Vol. 7, pp. 3-30). Bingley: Emerald.

Freeman, L. C. (1977). A set of measures of centrality based on betweenness. Sociometry, 40(1), 35-41.

Gibson, V. A., \& Lizieri, C. (2001). Friction and inertia: Business change, corporate real estate portfolios and the UK office market. Journal of Real Estate Research, $22(1 / 2), 59-79$

Gibson, V., \& Louargand, M. (2001). The workplace portfolio as contractual arrangements. In M. Bell \& M. Joroff (Eds.), The agile workplace: Supporting people and their work (pp. 34-51). Boston, MA: Gartner and MIT.

Gibler, K. M., Black, R. T., \& Moon, K. P. (2002). Time, place, technology and corporate real estate strategy, Journal of Real Estate Research, $24(3), 235-262$.

Goñi-Legaz, S., \& Ollo-López, A. (2015). Factors that determine the use of flexible work arrangement practices in Spain. Journal of Family and Economic Issues, 36, 463-476.

Gong, X., Jiang L., Yang H., \& Wu, F. (2013). Mapping intellectual structure: A cocitation analysis of food safety in CiteSpace II. Retrieved from http://cluster.ischool.drexel.edu/ cchen/courses/INFO633/12-13/gong.pdf

Handy, C. (1995). Trust and the virtual organisation. Harvard Business Review, 73(3), 40-50.

Haynes, B. P. (2008). Impact of workplace connectivity on office productivity. Journal of Corporate Real Estate, 10(4), $286-302$.

Heerwagen, J., Kelly, K., \& Kampschroer, K. (2016). The changing nature of organisations, work, and workplace. Retrieved from https://www.wbdg.org/resources/changing-nature-organizations-work-and-workplace

Helen, S. (2016). Making the most of new working practices. In StrategicRISK (pp. 27-29). London: Newsquest Specialist Media.

Hong, Y., Chan, D. W. M., Chan, A. P. C., \& Yeung, J. F. Y. (2012). Critical analysis of partnering research trend in construction journals. Journal of Management in Engineering, 28(2), 82-95.

Huselid, M. A. (1995). The impact of human resource management practices on turnover, productivity, and corporate financial performance. Academy of Management Journal, 38(3), 635-672.

Jayantha, W. M., Lau, S. S. Y., Chau, K. W., \& Ganesan, S. (2007). Influence of firm size, age and usage of IT on the growth of firms: Some evidence from Hong Kong's service sector. International Journal of Urban Sciences, 11(1), 42-56.

Jin, Y., Ji, S., Li, X., \& Yu, J. (2017). A scientometric review of hotspots and emerging trends in additive manufacturing. Journal of Manufacturing Technology Management, 28(1), 18-38.

Kelliher, C., \& Anderson, D. (2010). Doing more with less? Flexible working practices and the intensification of work. Human Relations, 63(1), 83-106.

Kim, M. C., \& Chen, C. (2015). A scientometric review of emerging trends and new developments in recommendation systems. Scientometrics, $104,239-263$.

Klochkov, Y. (2019, February). Analysis of the publication activity of university researchers. In AICAI'19. 2019 Amity International Conference on Artificial Intelligence (pp. 74-79). Los Alamitos, CA: IEEE.

Kossek, E. E., Pichler, S., Bodner, T., \& Hammer, L. B. (2011). Workplace social support and work-family conflict: A meta-analysis clarifying the influence of general and work-family-specific supervisor and organisational support. Personnel Psychology, 64(2), 289-313.

Lee, P.-C., \& Su, H.-N. (2010). Investigating the structure of regional innovation system research through keyword co-occurrence and social network analysis Innovation, 12(1), 26-40.

Leoni, R., Cristini, A., Labory, S., \& Gaj, A. (2001, June). New work practices in Italy. Adoption and performance effects. Paper presented at the International Conference on Organisational Designs, Management Styles and Firm Performance, Bergamo, Italy.

Lizieri, C. M. (2003). Occupier requirements in commercial real estate markets. Urban Studies, 40(5-6), 1151-1169.

Manning, C., \& Roulac, S. E. (2001). Corporate real estate research thought leadership continues to evolve. Journal of Real Estate Research, 22 (1/2), 1-6.

McEwan, A.-M., \& Ennals, R. (2005) Building social capital and regional innovation through Healthy Working Centres: An investigation in the South East of England. AI \& Society, 19, 348-361.

McOrmond, T. (2004). Changes in working trends over the past decade. Labour Market Trends, 112(1), 25-36.

Millward, N. (1992). Workplace industrial relations in transition: The ED/ESRC/PSI/ACAS surveys. Aldershot: Dartmouth.

$\mathrm{Ng}$, C. F. (2016). Public spaces as workplace for mobile knowledge workers. Journal of Corporate Real Estate, 18(3), 209-223.

Nijp, H. H., Beckers, D. G. J., Geurts, S. A. E., Tucker, P., \& Kompier, M. A. J. (2012). Systematic review on the association between employee worktime control and work-non-work balance, health and wellbeing, and job-related outcomes. Scandinavian Journal of Work, Environment \& Health, 38(4), $299-313$.

Olawumi, T. O., \& Chan, D. W. M. (2018). A scientometric review of global research on sustainability and sustainable development. Journal of Cleaner Production, $183,231-250$.

Oliner, S. D., \& Sichel, D. E. (2000). The resurgence of growth in the late 1990s: Is information technology the story? Journal of Economic Perspectives, 14(4), 3-22.

Ollo-Lopez, A., Bayo-Moriones, A., \& Larraza-Kintana, M. (2010). The relationship between new work practices and employee effort. Journal of Industrial Relations, $52(2), 219-235$.

Ollo-López, A., Bayo-Moriones, A., \& Larraza-Kintana, M. (2011). The impact of country-level factors on the use of new work practices. Journal of World Business, 46(3), 394-403.

Osterman, P. (1994). How common is workplace transformation and who adopts it? ILR Review, 47(2), 173-188.

Pollack, J., \& Adler, D. (2015). Emergent trends and passing fads in project management research: A scientometric analysis of changes in the field. International Journal of Project Management, 33(1), 236-248.

Ramirez, M., Guy, F., \& Beale, D. (2007) Contested resources: Unions, employers, and the adoption of new work practices in US and UK telecommunications. British Journal of Industrial Relations, 45(3), 495-517.

Schøne, P. (2009). New technologies, new work practices and the age structure of the workers. Journal of Population Economics, $22,803-826$.

Skinner, N., \& Chapman, J. (2013). Work-life balance and family friendly policies. Evidence Base, (4), 1-25.

Smith, S., \& Ward, V. (2015). The role of boundary maintenance and blurring in a UK collaborative research project: How researchers and health service managers made sense of new ways of working. Social Science \& Medicine, 130, 225-233.

Tucker, D. A., Hendy, J., \& Barlow, J. (2014). When infrastructure transition and work practice redesign collide. Journal of Organizational Change Management, 27(6), 955-972.

Waterhouse, P., Wilson, B., \& Ewer, P. (1999). The changing nature and patterns of work and implications for VET: Review of research. Kensington Park: NCVER.

Wohlers, C., \& Hertel, G. (2018). Longitudinal effects of activity-based flexible office design on teamwork. Frontiers in Psychology, 9, 2016.

Yin, R. K. (2009). Case study research: Design and methods (4th ed.). Thousand Oaks, CA: Sage.

Zhao, X. (2017). A scientometric review of global BIM research: Analysis and visualisation. Automation in Construction, 80, 37-47.

Zhou, Z., Goh, Y. M., \& Li, Q. (2015). Overview and analysis of safety management studies in the construction industry. Safety Science, 72, 337-350. 